\title{
Characterization of ER-mitochondria contact sites using cryo-CLEM
}

Reza Paraan ${ }^{1}$, Victoria Hewitt ${ }^{2}$, Yusuke Hirabayashi ${ }^{3}$, Franck Polleux ${ }^{2}$, Clint Potter ${ }^{4}$ and Bridget Carragher 4

${ }^{1}$ New York Structural Biology Center, United States, ${ }^{2}$ Columbia University, United States, ${ }^{3}$ University of Tokyo, United States, ${ }^{4}$ New York Structural Biology Center, New York, New York, United States

Several vital physiological processes, such as phospholipid and $\mathrm{Ca} 2+$ exchange, mitochondrial biogenesis, autophagy, and intracellular trafficking rely on the contact sites between the endoplasmic reticulum and mitochondria (Kornmann et al., 2009; Hirabayashi et al., 2017). These processes are affected in neurodegenerative disorders such as Alzheimer's (Area-Gomez and Schon, 2016), Parkinson's, amyotrophic lateral sclerosis (ALS), and frontotemporal dementia (FTD) (Paillusson et al., 2016). However, it is not clear how these disorders can affect multiple seemingly unrelated physiological pathways. ER-mitochondria contact sites are emerging as candidates in understanding the origins of the pathophysiology of these disorders. The contact sites are small sporadic areas where the two organelles are less than 30 nanometers apart. There are specialized protein complexes that reside at these sites. This results in a complex molecular landscape that is not fully understood. Several protein complexes have been proposed as the tethers that bridge these membranes. Tethers are comprised of ER-resident proteins, as well as mitochondrial outer membrane proteins, and in some cases soluble intermediate proteins. Here, the molecular composition and architecture of one of these tethers is studied in eukaryotic cells with endogenous fluorescence tags. One ER-resident protein, PDZD8, and one interacting partner, a mitochondrial outer membrane protein, are studied in vivo. Further, the structure of these macromolecular complexes is explored using cryo-EM. With state-of-the-art cryo-correlative light and electron microscopy, the distribution of the tethers as well as their structural mechanisms of mediating contact are uncovered and characterized.

Division of work:

In vivo studies are carried out in Franck Polleux and Yusuke Hirabayashi's lab, in Columbia University and the University of Tokyo, respectively.

Cryo-EM is done in Simons Electron Microscopy Center (SEMS) at New York Structural Biology Center (NYSBC), under the supervision of Bridget Carragher and Clint Potter.

\section{References}

Area-Gomez E, Schon EA. Mitochondria-associated ER membranes and Alzheimer disease. Curr Opin Genet Dev. 2016 Jun;38:90-96. doi: 10.1016/j.gde.2016.04.006. Epub 2016 May 25. PMID: 27235807; PMCID: PMC5390896.

Hirabayashi, Y., Kwon, S. K., Paek, H., Pernice, W. M., Paul, M. A., Lee, J., ... \& Polleux, F. (2017). ERmitochondria tethering by PDZD8 regulates Ca2+ dynamics in mammalian neurons. Science, 358(6363), 623-630.

Kornmann B, Currie E, Collins SR, Schuldiner M, Nunnari J, Weissman JS, Walter P. An ERmitochondria tethering complex revealed by a synthetic biology screen. Science. 2009 Jul 
24;325(5939):477-81. doi: 10.1126/science.1175088. Epub 2009 Jun 25. PMID: 19556461; PMCID: PMC2933203.

Paillusson S, Stoica R, Gomez-Suaga P, Lau DHW, Mueller S, Miller T, Miller CCJ. There's Something Wrong with my MAM; the ER-Mitochondria Axis and Neurodegenerative Diseases. Trends Neurosci. 2016 Mar;39(3):146-157. doi: 10.1016/j.tins.2016.01.008. Epub 2016 Feb 15. PMID: 26899735; PMCID: PMC4780428. 